\title{
Assessment of right and left ventricular function in terms of force development with gestational age in the normal human fetus
}

Cardiovascular Division, Department of Medicine, Department of Radiology, Department of Obstetrics and Gynaecology, Brigham and Women's Hospital, Harvard Medical School, Boston, Massachusetts, USA M St John Sutton T Gill

T Plappert

D H Saltzman P Doubilet

Correspondence to Dr Martin St John Sutton, Cardiovascular Division, Royal Brompton National Heart and Lung Hospital, Sydney Street, London SW3

Accepted for publication 29 April 1991

\author{
Martin St John Sutton, Tom Gill, Ted Plappert, Daniel H Saltzman, Peter Doubilet
}

\begin{abstract}
Objective-To assess right and left ventricular systolic function in normal human fetuses by cross sectional Doppler echocardiography to calculate the force developed by myocardial shortening.

Design-Cross sectional echocardiographic images of the aorta and pulmonary arteries were obtained prospectively in order to measure great vessel diameters and calculate their cross sectional areas. Doppler velocity signals were recorded from the proximal aorta and the proximal pulmonary artery and digitised to obtain peak velocity, acceleration time, flow velocity time integral during acceleration, and the flow velocity time integral for the whole of ejection. Right and left ventricular force development was estimated by Newton's equation in which force is defined as the product of mass and acceleration.
\end{abstract}

Patients-58 normal human fetuses at a gestational age of from 20 to 42 weeks.

Results-The cross sectional area of the pulmonary artery was $20 \%$ greater than that of the aorta. Aortic acceleration time was longer than that in the pulmonary artery, and peak blood flow velocity in the aorta was consistently greater than that in the pulmonary artery. Right ventricular stroke volume was significantly greater than left ventricular stroke volume. The force developed by the right and left ventricles was, however, similar throughout the gestational period studied, increasing tenfold from 20 weeks' gestation to term $(r=0.74, \quad p<0.0001 ; \quad r=0.75$, p $<0.0001)$ respectively.

Conclusion-The development of right and left ventricular force in the human fetus is similar in spite of the greater volume handled by the right ventricle. This index of ventricular performance does not require calculation of ventricular volume and because it varies independently of ventricular geometry and heart rate it should prove useful in assessing cardiac function in the normal human fetus and in fetuses with ventricular dysfunction.

Assessment of right and left ventricular function in terms of ejection fraction is not feasible in the human fetus because ventricular cham- ber volumes are too small to quantify accurately. Currently the only available measures of ventricular function in the human fetus are percent systolic shortening of the right and left ventricular short axis diameters and estimation of ventricular stroke volumes with cross sectional Doppler echocardiography. ${ }^{1-5}$ Often it is not possible to assess ventricular systolic shortening because the ultrasound beam cannot be correctly aligned to pass through the right and left ventricular diameters. Moreover, although stroke volume can be calculated in most fetuses, it varies inversely with heart rate $^{67}$ and does not provide information regarding the efficiency of ventricular emptying.

A non-invasive method of assessing right and left ventricular function would be useful not only in normal human fetuses but more importantly in fetuses with ventricular dysfunction caused by Rhesus incompatibility, hypoxaemia, and recurrent tachycardias. ${ }^{8}$ In addition, such a technique would be of value for early detection of the adverse cardiovascular effects of tocolytic $\beta$ sympathomimetic agents which have become the mainstay treatment for prevention of premature birth. ${ }^{9}$

We describe a method of measuring right and left ventricular function in the human fetus in terms of myocardial force development that does not require estimation of ventricular volumes and is independent both of ventricular configuration and cardiac cycle length over the range of heart rates encountered.

This study had three aims: first to calculate the force developed by the two ventricles, second to assess the magnitude of changes in ventricular performance with gestational age, and third to determine whether there were any systematic differences between the two ventricles.

\section{Patients and methods}

STUDY POPULATION

We studied 58 normal fetuses whose mothers were recruited from patients referred for routine ultrasound examination. The indications for ultrasound examination were estimation of gestational age and placental location. Mothers with diabetes, hypertension, eclampsia, Rhesus incompatibility, and multiple gestations were excluded. Doppler echocardiograms were obtained from fetuses ranging in gestational age from 20 to 42 weeks. The study protocol was approved by the Brigham and Women's Hospital Human Subjects Com- 
Figure 1 Doppler velocity signal from the aorta shown with schematic electrocardiogram. $A T$

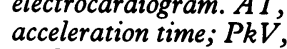
peak velocity; $F V I_{A T}$ blood velocity integral during acceleration.

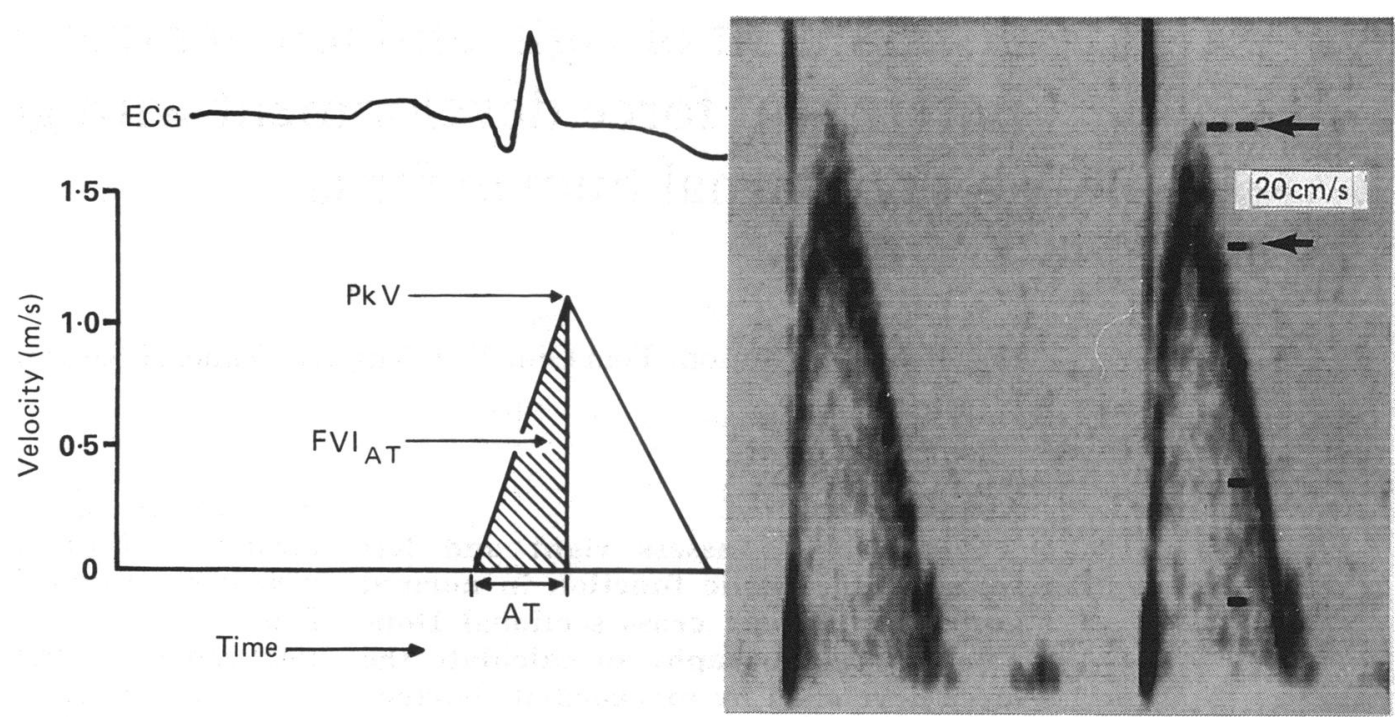

mittee of Harvard Medical School and written informed consent was obtained from each mother.

\section{DATA ACQUISITION}

Cross sectional Doppler echocardiographic studies were performed with a Hewlett Packard ultrasound system and a $3.5 \mathrm{MHz}$ or more often a $5.0 \mathrm{MHz}$ transducer. Each mother rested in the recumbent position for 15 minutes then a transducer was placed on her abdomen and the fetal heart was located using the fetal spine and liver as easily recognisable landmarks. A systematic cross sectional echocardiographic examination of the fetal heart was performed to exclude any structural abnormality and to confirm the presence of normal atrial situs, concordant atrioventricular connections, concordant ventriculoarterial connections, and normal cardiac chamber sizes and functions. The great arteries were identified by their characteristic pattern of major branches.

High quality cross sectional echocardiographic images of the aorta and pulmonary artery were obtained with the ultrasound beam aligned as orthogonally as possible with the axial plane of the vessels to measure their diameters. Doppler blood flow velocity signals were recorded from the main pulmonary artery and from the ascending aorta immediately distal to the respective semilunar valves. The pulsed wave Doppler sample volume was positioned within the lumen of the pulmonary artery and aorta and its size adjusted to sample velocities from the whole of the flow stream. The insonating beam was aligned as close as possible to parallel the direction of blood flow. The flow direction cursor was used to measure the angle of incidence between the ultrasound beam and the direction of blood flow, and Doppler velocity signals were only recorded when this was less than $20^{\circ}$. We chose $20^{\circ}$ as a cut off because flow velocity varies inversely with the cosine of the angle of incidence, and velocity measured at an angle of $20^{\circ} \mathrm{C}$ underestimates true velocity by no more than $8 \%$. Doppler signals with maximum velocity were recorded during periods of apnoea because fetal respiratory efforts can alter velocity amplitude. ${ }^{111}$
Heart rate was measured from the Doppler velocity signals obtained from the great arteries.

The gestational age of each fetus was assessed from the first day of the last menstrual period and corroborated by ultrasound measurements of biparietal cephalic diameter..$^{1213}$

\section{DATA ANALYSIS}

Cross sectional echocardiographic images of the aorta and pulmonary artery were transferred from half inch videotape to the digital disc of a Frieland Medical off-line computer analysis system. The aortic and pulmonary artery diameters were measured at the level of the annulus immediately distal to the semilunar valves at the time of maximum leaflet separation. The gain controls of the ultrasonoscope were adjusted to minimise "blooming" of echoes from the vessel walls. Measurements were made with an electronic cursor from a minimum of five separate cardiac cycles and rounded off to the nearest millimetre. Mean values were calculated for each vessel diameter.

Cross sectional areas of the pulmonary artery and of the aorta were calculated assuming a circular cross section CSA $=\pi(\mathrm{D} / 2) .{ }^{2}$ Doppler velocity signals recorded from the aorta and pulmonary artery from a minimum of three consecutive cardiac cycles during periods of fetal apnoea were transferred from videotape to the hard disc of the computer analysis system. The velocity spectral envelopes with the maximum velocities were digitised through the middle of the densest portion of the spectral envelope. The following measurements were made from the pulmonary artery and the aorta: (a) Peak velocity (pkV); (b) acceleration time (AT), defined as the time from the initial increase in velocity to the time of peak velocity. (fig 1); (c) the flow velocity time integral during acceleration $\left(\mathrm{FVI}_{\mathrm{AT}}\right)$, which is the area under the velocity spectral envelope up to the time of peak velocity (fig 1 ); and $(d)$ total velocity time integral FVI for the whole of ejection.

The force developed by each right and left ventricular contraction accelerated a column of blood into the pulmonary artery and aorta respectively and represented transfer of the 


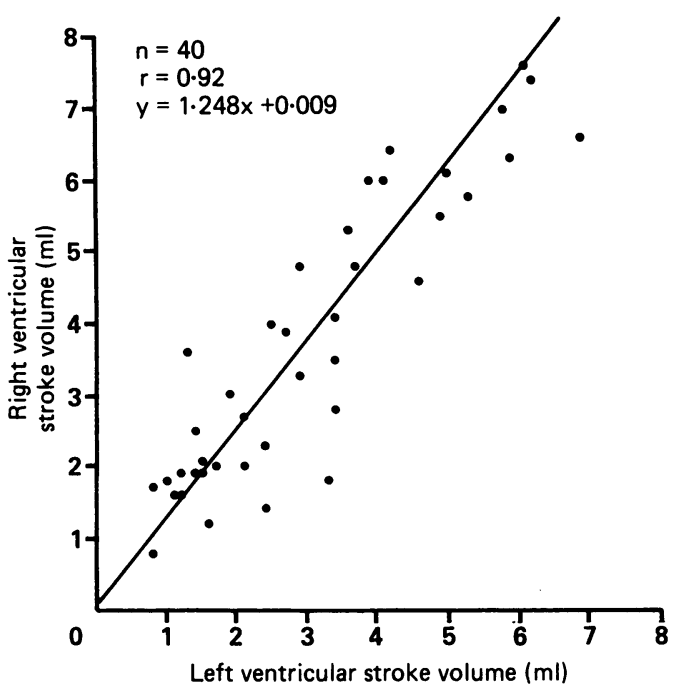

Figure 2 Comparison of right and left ventricular stroke volumes showing that for the population as a whole the right ventricular stroke volume was greater than left ventricular stroke volume.

energy of myocardial shortening to work done on the pulmonary and systemic circulations. We used Newton's equation to estimate the force (that is, the product of mass and acceleration $(\mathrm{F}=\mathrm{ma})){ }^{14}$ The mass component of the force equation was the mass of blood accelerated into the arteries, which was calculated as the product of the density of blood $(1.055)$, the cross sectional area of the great artery (CSA), and the flow velocity time integral during acceleration $\left(\mathrm{FVI}_{\mathrm{AT}}\right)$ (fig 1). The acceleration component of the force equation was estimated as the peak velocity divided by the acceleration time (pkV/AT)-thus force $\mathrm{F}=\left(1.055 \times \mathrm{CSA} \times \mathrm{FVI}_{\mathrm{AT}}\right) \times(\mathrm{pkV} / \mathrm{AT})$.

Right and left ventricular stroke volumes (SV) were calculated as the products of the

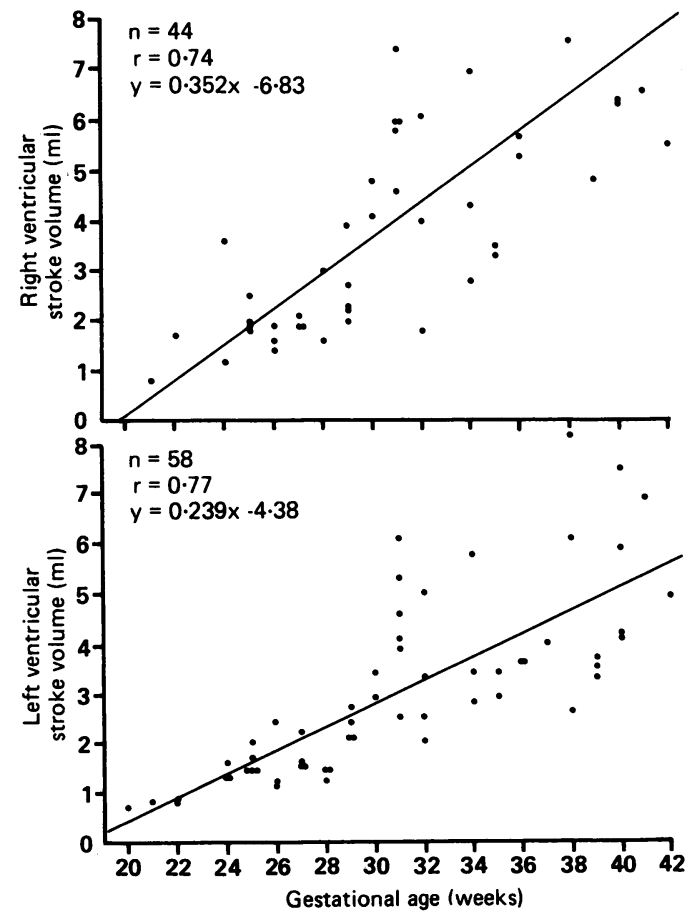

Figure 3 Plot showing a linear increase in right and left ventricular stroke volumes with gestational age.

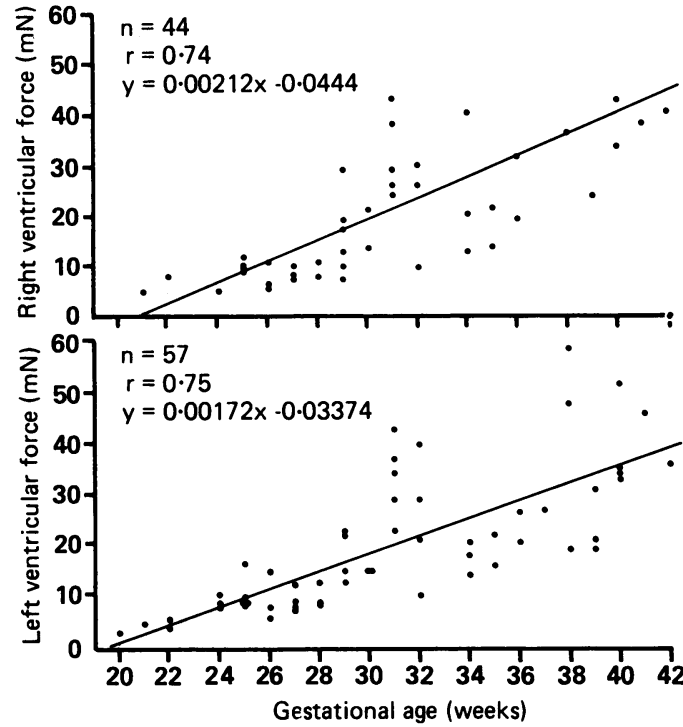

Figure 4 Plots showing a linear increase in right and left ventricular force with gestational age.

cross sectional areas of the great arteries (CSA) and the flow velocity time integrals (FVI) of the Doppler signals obtained from the respective great arteries, so that $\mathrm{SV}=(\mathrm{FVI} \times \mathrm{CSA})$.

\section{STATISTICAL ANALYSIS}

Student's $t$ test was used to assess the significance of differences between right and left ventricular force development, stroke volumes, peak velocities, aortic and pulmonary cross sectional areas, and acceleration times. Values are given as mean (1 SD).

We assessed changes in myocardial force development and stroke volume with gestational age and changes in right and left ventricular force and stroke volumes with gestational age by regression analysis using Pearson's method.

\section{Results}

Pulmonary artery diameters were larger than aortic diameters throughout the period of gestation studied, so that the mean cross sectional

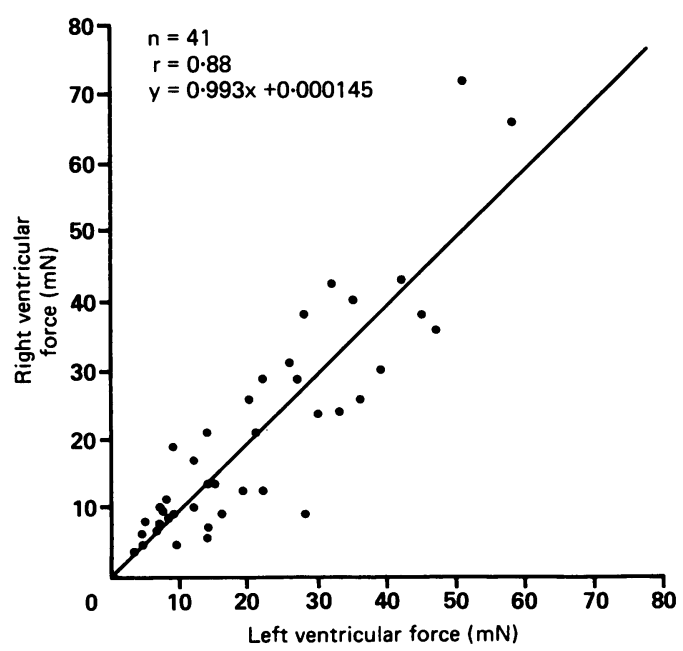

Figure 5 Plot comparing right and left ventricular force development and showing similar force development in the right and left ventricles. 
area of the pulmonary artery was $20 \%$ greater than that of the aorta $\left(0.31(0.16) \mathrm{cm}^{2} v 0.39\right.$ $\left.(0.20) \mathrm{cm}^{2}\right)(\mathrm{p}<0.01)$. The blood flow velocities in the aorta were consistently greater than velocities in the pulmonary artery (mean $0.88(0.14) \mathrm{m} / \mathrm{s} v 0.78(0.13) \mathrm{m} / \mathrm{s}$.) $(\mathrm{p}<0.0001)$. However, neither the aortic nor the pulmonary artery peak velocities varied significantly with heart rate $(\mathrm{r}=-0.01$, $r=+0.04$ respectively).

The mean ventricular stroke volume ejected by the right ventricle was $24 \%$ greater than that ejected by the left ventricle for the population as a whole $(4.1(2.6) \mathrm{ml} v 3.3(2.0) \mathrm{ml})$ $(\mathrm{p}<0.01)$ (fig 2). Both right and left ventricular stroke volumes increased progressively with gestational age (fig 3 ). Heart rate declined from a mean of 144 beats per minute at 20 weeks to a mean of 129 beats per minute at 42 weeks. The acceleration time in the aorta was significantly longer than that in the pulmonary artery $(44.6(6.9) \mathrm{ms} \quad v \quad 39.4(10.6) \mathrm{ms})$ $(\mathrm{p}<0.001)$.

The myocardial force developed by the right and left ventricles increased more than tenfold from 20 weeks' gestation to term, $(r=0.74$, $\mathrm{p}<0.0001 ; \mathrm{r}=0.75, \mathrm{p}<0.0001$ ) (fig 4). The force developed by the right ventricle was similar to that developed by the left ventricle throughout the period of gestation studied (fig 5). The force developed by both ventricles varied independently of heart rate $(r=-0.12$ and $\mathrm{r}=-0 \cdot 11$ ).

\section{REPRODUCIBILITY}

The reproducibility of measurements of great vessel diameters in the human fetus has already been reported and the consequences of errors in diameter measurement on volume flow calculations derived from them have been estimated. ${ }^{15}$

\section{Discussion}

The lack of a simple measure of ventricular performance in the human fetus prompted us to develop a non-invasive technique to measure the force developed by both right and left ventricles. We chose to measure ventricular force because it can be defined in physical terms and because it relates directly to myocardial systolic shortening. Importantly, myocardial systolic shortening, force development, and the force-velocity relations in the fetal myocardium differ from those in the adult myocardium. ${ }^{16}$ These differences in myocardial mechanics are determined by the different enzyme kinetics of the myosin ATP'ase on the fetal and adult myosin isoforms. ${ }^{1718}$

We estimated force as the product of mass and acceleration as defined in Newton's equation. This required simple measurements of blood flow velocity signals and great artery diameters, all of which have been reported individually in several previous studies. ${ }^{451920}$

The concept of force development was attractive for several reasons. First, it did not require any assumptions regarding ventricular geometry or size. Second, it did not need measurement of ventricular volumes. Third, it was not affected by variation in heart rate over the physiological range encountered. Fourth, it represented an estimation of the energy transferred from right and left ventricular myocardial shortening to work done by accelerating blood into the pulmonary and systemic circulations respectively.

The cross sectional areas of the pulmonary artery were consistently greater than those of the aorta throughout the period of gestation studied, but the reverse was true of the peak blood flow velocities. The mean stroke volume ejected by the right ventricle was more than $20 \%$ greater than that ejected by the left ventricle for the population as a whole; this result accorded with previous reports. ${ }^{45}$ Acceleration time was longer in the aorta than in the pulmonary artery, as has been previously described, ${ }^{21}$ and varied inversely with heart rate. Acceleration time was longer in the aorta, whereas in normal children and adults acceleration time in the pulmonary artery is longer, as is the right ventricular ejection time.

The main finding in this study was that the force developed by the right and left ventricles was similar throughout the latter part of the second and third trimesters even though the right ventricle ejected a significantly greater stroke volume than did the left ventricle. The myocardial force in both ventricles increased more than tenfold from 20 weeks to term and this increase paralleled the increase in heart weight and total body weight, ${ }^{322}$ indicating that force increases as myocardial mass increases with fetal growth. The variability in force development in the third trimester most probably relates to the variability in fetal body habitus and therefore myocardial mass.

The similarity between the force developed by the two ventricles was initially surprising in view of the significantly different stroke volumes. Myocardial systolic shortening in the intact ventricle is determined by several factors, important among which are ventricular chamber geometry and ventricular loading conditions or afterloads. ${ }^{2324}$ Calculation of right and left ventricular afterloads requires the measurement of chamber diameters, wall thicknesses, and ventricular systolic pressures. Left and right ventricular diameters, wall thicknesses, and free wall weights were found to be similar in the human fetus when they were measured in vivo by ultrasound and in vitro in necropsy specimens, ${ }^{322}$ indicating similar right and left ventricular chamber geometry. However, right and left ventricular systolic pressures cannot be measured in the human fetus and therefore ventricular afterloads cannot be quantified. In the fetal lamb, which has been used extensively as an experimental model, right and left ventricular systolic pressures are equal, ${ }^{25}{ }^{26}$ and there is little reason to believe they are different in the human fetus. Thus ventricular afterload, which is the force that the myocardium must overcome to eject blood into the pulmonary or systemic circulations, must be the same in the two ventricles. Because afterload varies inversely with ventricular fractional shortening further evidence that right and left ventricular afterloads are equivalent is provided by the observation that 
fractional shortening is similar in the right and left ventricles. Since right and left ventricular chamber geometries, afterloads, and myocardial shortening are all closely similar, it is easier to understand how the two ventricles develop the same amount of myocardial force even though their respective volume handling is different. The right ventricle ejects a larger stroke volume than the left ventricle, but does so at a lower velocity than the left ventricle.

We describe a non-invasive method of assessing right and left ventricular performance in terms of myocardial force in the human fetus. The study showed that the force developed by the right and left ventricles is similar and increases more than tenfold from 20 weeks to term. We submit that this quantitative method of assessing ventricular function may be of clinical value not only in the normal fetus but also in monitoring fetuses with impaired ventricular function.

1.Sahn DJ, Lange LW, Allen HD, Goldberg SJ, Anderson C, Giles J, et al. Quantitative real-time cross-sectional echocardiography in the developing normal human fetus and newborn. Circulation 1980;62:588-97.

2 Allan LD, Joseph MC, Boyd EGCA, Campbell S, Tynan M. $M$-mode echocardiography in the developing human fetus. Br Heart J 1982;47:573-83.

3 St John Sutton M, Gewitz MH, Shah B, Cohen A, Reichek $\mathrm{R}, \mathrm{Gabbe} S$, et al. Quantitative assessment of growth and function of the cardiac chamber in the normal human fetus: a prospective longitudinal study. Circulation 1984;69:645-54.

4 Reed KL, Meijboom EJ, Sahn DJ, Scagnelli SA, ValdesCruz L M, Shenker L. Cardiac Doppler flow velocities in human fetuses. Circulation 1986;73:41-6.

5 Kenny JF, Plappert T, Doubilet P, Saltzman DH, Cartier $M$, Zollars L, et al. Changes in intracardiac blood flow velocities and right and left ventricular stroke volumes with gestational age in the normal human fetus: a prospec-
tive Doppler echocardiographic study. Circulation tive Doppler ech
1986;74:1208-16.

6 Anderson PAW, Glick KL, Killam AP, Mainwaring RD. The effect of heart rate in utero on left ventricular output in the fetal sheep. J Physiol (Lond) 1986;372:557-73.

7 Kenny J, Plappert T, Doubilet P, Saltzman D, St John Sutton $M$. Effects of heart rate on ventricular size, stroke volume, and output in the normal human fetus; a prospective Doppler echocardiographic study. Circulation
1987;76:52-8.
8 Kleinman CS, Donnerstein RL, Devore GR, Jaffe CC, Lynch DC, Berkowitz RL, et al. Fetal echocardiography for evaluation of in utero congestive heart failure. $N \mathrm{Engl} J$ Med 1982;306:568-75.

9 Katz VL, Seeds JW. Fetal and neonatal cardiovascular complications from B-sympathomimetic therapy for tocolysis. Am J Obstet Gynecol 1989;161:1-4.

10 Gill RW. Measurement of blood flow by ultrasound: accuracy and sources of error. Ultrasound Med Biol 1985;11:625-41.

11 Griffin D, Cohen-Overbeek T, Campbell S. Fetal Uteroplacental blood flow. Clin Obstet Gynecol 1983;10:565-74.

12 Campbell S, Newman GB. Growth of the fetal biparietal diameter during normal pregnancy. J Obstet Gynecol Br Commonwealth 1971;73:513-9.

13 Kurtz AB, Warner RJ, Dershaw DD, Rubin CS, ColeBeuglet C, Goldberg BB. Analysis of biparietal diameters as an accurate indicator of gestational age. $J$ Clin as an accurate indicator

14 Isaaz K, Ethevenot G, Admant P, Brembilla B, Pernot C. A new Doppler method of assessing left ventricular ejection force in chronic congestive heart failure. Am J Cardio 1989;64:81-7.

15 St John Sutton $M$, Theard $M$, Bhatia SJS, Plappert $T$, Doubilet $P$, Saltzman DH. Changes in placental blood flow in the normal human fetus with gestational age. Pediat Res 1990;28:383-8.

16 Friedman WF. The intrinsic physiologic properties of the developing heart. Prog Cardiovasc Dis 1972;15:87-111.

17 Carey RA, Bove AA, Coulson RL, Spann JF. Correlation between cardiac muscle myosin ATP'ase activity and velocity of muscle shortening. Biochem Med 1979;21: 235-45.

18 Schwartz K, Le Carpentier Y, Martin JL, Lompre AM, Mercadier JJ, Swinghedauw B. Myosin isoenzyme distribution correlates with speed of myocardial contraction. tribution correlates with speed of m

19 Angelini A, Allan LD, Anderson RH, Crawford DC, Chit SK, Ho SY. Measurements of the dimensions of the aortic and pulmonary pathways in the human fetus: a correlative echocardiographic and morphometric study. Br Heart $J$ 1988;60:221-6.

20 Cartier MS, Davidoff A, Warneke LA, Hirsch MP, Bannon $S$, St John Sutton M, et al. The normal diameter of the fetal aorta and pulmonary artery: Echocardiographic evaluation in utero. AJR 1987;149:1003-7.

21 Machado MVL, Chita SC, Allan LD. Acceleration time in the aorta and pulmonary artery measured by Doppler echocardiography in the midtrimester normal human fetus. Br Heart J 1987;58:15-8.

22 St John Sutton M, Raichlen JS, Reichek N, Huff DS. Quantitative assessment of right and left ventricular growth in the human fetal heart: a pathological study. Circulation 1984;70:935-41.

23 Weber KT, Janicki JS. The dynamics of ventricular contraction, force, length and shortening. Fed Proc 1980;39: tion, force,

24 Borow KM, Green LH, Grossman W, Braunwald E. Left ventricular end-systolic stress shortening and stresslength relationships in humans: normal values and sensitivity to inotropic state. Am J Cardiol 1982;50:1301-8.

25 Rudolph AM. Organization and control of the fetal circulation. In: Jones C, Nathaniels PW, eds: The physiological development of the fetus and newborn. London: Academic Press, 1985:343-53.

26 Rudolph AM, Heymann MA. Fetal and neonatal circulation and respiration. Annu Rev Physiol 1974;19:187-201. 